\title{
Erratum to: GABA content within medial prefrontal cortex predicts the variability of fronto-limbic effective connectivity
}

\author{
Stefano Delli Pizzi ${ }^{1,2,3} \cdot$ Piero Chiacchiaretta $^{1,2} \cdot$ Dante Mantini $^{4,5,6} \cdot$ \\ Giovanna Bubbico $^{1,2} \cdot$ Richard A. Edden $^{7,8}$ - Marco Onofrj ${ }^{1,3}$ - Antonio Ferretti ${ }^{1,2}$. \\ Laura Bonanni ${ }^{1,3}$ (i)
}

Published online: 8 June 2017

(c) Springer-Verlag Berlin Heidelberg 2017

\section{Erratum to: Brain Struct Funct \\ DOI 10.1007/s00429-017-1399-x}

The second co-author name has been misspelled in the original publication of the article. The correct name should be "Piero Chiacchiaretta".

The Original article has been updated accordingly.

The online version of the original article can be found under doi:10.1007/s00429-017-1399-x.

Laura Bonanni

1.bonanni@unich.it

1 Department of Neuroscience, Imaging and Clinical Sciences, University "G. d'Annunzio" of Chieti-Pescara, Via dei Vestini, 66100 Chieti, Italy

2 Institute for Advanced Biomedical Technologies (ITAB), "G. d'Annunzio" University, Chieti-Pescara, Italy

3 Center of Aging Sciences and Translational Medicine (CeSI-MeT), University "G. d'Annunzio" of Chieti-Pescara, Chieti, Italy

4 Research Centre for Motor Control and Neuroplasticity, KU Leuven, Heverlee, Belgium

5 Department of Health Sciences and Technology, Neural Control of Movement Lab, Zurich, Switzerland

6 Department of Experimental Psychology, Oxford University, Oxford, UK

7 Russell H. Morgan Department of Radiology,

The Johns Hopkins University School of Medicine, Baltimore, MD, USA

8 F.M. Kirby Center for Functional MRI, Kennedy Krieger Institute, Baltimore, MD, USA 